\title{
A REVIEW OF OCULAR FUNGAL INFECTIONS OTHER THAN KERATOMYCOSIS: THE GLOBAL AND INDIAN SCENARIO
}

\author{
Somnath Sarkar1, Tapas Kumar Bhattacharya², Atanu Roy33, Kumarjyoti Ghosh ${ }^{4}$ \\ ${ }^{1}$ Associate Professor, Department of Ophthalmology, MGM Medical College \& LSK Hospital, Bihar. \\ ${ }^{2}$ Associate Professor, Department of Microbiology, MGM Medical College \& LSK Hospital, Bihar. \\ ${ }^{3}$ Professor and HOD, Department of Microbiology, MGM Medical College \& LSK Hospital, Bihar. \\ ${ }^{4}$ Assistant Professor, Department of Microbiology, MGM Medical College \& LSK Hospital, Bihar.
}

\section{ABSTRACT}

Fungal ocular infections are common in the developing countries having hot, humid climate and poor health educational status. Trauma with vegetable matter, indiscriminate use of steroid in over-the-counter topical preparations and poor penetrability of antifungal agents make these disease entities common and rather difficult to treat. Besides corneal infections by fungi (Keratomycosis), there are other ocular sites which are involved: eyelids, conjunctiva, uvea, vitreous, retina, orbit and ocular adnexa. Some of these infections may be life-threatening and/or may lead to blindness. This review summarises the clinical features of the ocular involvement, discusses the risk factors for the infections and also explains about therapeutic and preventive measures to combat the infections. It also gives an idea of the current global and Indian disease spectrum.

\section{KEYWORDS}

Non-keratitic Ocular Infection, Exogenous Fungal Infection, Endogenous Fungal Infection, Systemic Antifungal Drugs, Topical Antifungal Treatment, Rhino-Orbito-Cerebral Fungal Infection, Fungal Endophthalmitis.

HOW TO CITE THIS ARTICLE: Sarkar S, Bhattacharya TK, Roy A, et al. A review of ocular fungal infections other than keratomycosis: the Global and Indian scenario. J. Evolution Med. Dent. Sci. 2016;5(49):3168-3172, DOI: 10.14260/jemds/2016/734

\section{INTRODUCTION}

In the 1980s systemic fungal infection has emerged as a major cause of human disease mainly affecting hospitalised patients, particularly post-surgical, those with serious underlying illness; immuno-compromised patients and patients treated in Intensive Care Units. Indian studies and statistics also indicate that the incidence of systemic fungal infection is on the rise and is associated with increased morbidity and mortality.(1)

Fungal ocular infections are common in the developing countries with hot humid climate. They are also the most difficult infections to treat in view of the rather poor penetration of antifungal agents.(2) Long-term use of antifungal agents also gives rise to various systemic toxicities.

Fungal corneal ulcers represent the majority of cases of ocular fungal infections. Trauma with vegetable matter and indiscriminate use of over-the-counter topical steroid preparations are the main causes of development of fungal corneal ulcers. (2) Candida infection is common in diabetics and in the immunosuppressed patients. However, it has been seen that fungal disease may affect the eyes elsewhere other than that of the cornea.

Financial or Other, Competing Interest: None.

Submission 27-04-2016, Peer Review 27-05-2016,

Acceptance 02-06-2016, Published 20-06-2016.

Corresponding Author:

Dr. Somnath Sarkar,

Satabdi Apartment,

Flat-4, 333/D, Jessore Road, Kolkata-700089,

P.O. Lake Town, West Bengal.

E-mail: som29sarkar@gmail.com

DOI: $10.14260 /$ jemds/2016/734

\begin{tabular}{|l|l|l|}
\hline \multicolumn{1}{|c|}{ Yeasts } & \multicolumn{1}{|c|}{ Moulds } & \multicolumn{1}{|c|}{$\begin{array}{c}\text { Pneumocystis } \\
\text { Jirovecii in HIV } \\
\text { Patients }\end{array}$} \\
\hline \multicolumn{1}{|c|}{ Candida species } & Aspergillus species & \\
\hline $\begin{array}{l}\text { C. albicans, C. } \\
\text { glabrata, C. } \\
\text { parapsilosis, } \\
\begin{array}{l}\text { C. tropicalis, C. } \\
\text { krusei, C. } \\
\text { guilliermondii }\end{array}\end{array}$ & $\begin{array}{l}\text { A. fumigatus, A. } \\
\text { flavus, } \\
\text { A. niger, A. tereus }\end{array}$ & \\
\hline $\begin{array}{l}\text { Cryptococcus } \\
\text { neoformans }\end{array}$ & $\begin{array}{l}\text { Zygomycetes } \\
\text { (Mucormycetes) }\end{array}$ & \\
\hline $\begin{array}{l}70 \% \text { to } 90 \% \text { cases } \\
\text { of invasive } \\
\text { infection }\end{array}$ & $\begin{array}{l}10 \% \text { to 20\% cases of } \\
\text { invasive infection }\end{array}$ & \\
\hline
\end{tabular}

Table 1: Agents causing Opportunistic Fungal Infection

The affections in the eye other than that of the cornea have been discussed below.

\section{LIDS}

The lids may be affected in diabetic or immunosuppressed patients. In patients with poor personal hygiene, in patients with chronic epiphora and in patients with excessive facial sweating, fungal infections may occur. Pityriasis versicolor and Tinea corporis may occur in these patients. Itching with white discoloration or lid oedema may occur. The diagnosis is mainly clinical. Skin scrapings are examined after $10 \%$ to $20 \%$ potassium hydroxide mount under the compound microscope. This clinches the diagnosis as fungal elements are seen. The treatment is mostly with clotrimazole cream. Levocetirizine may be given to control the itching. The patients must be given proper health education regarding maintenance of personal hygiene. The systemic diseases such as diabetes mellitus must be controlled. If the lesions prove refractory to topical therapy, oral azoles can be added. 


\section{Conjunctiva}

Although various fungi can often be recovered from the conjunctiva, fungal conjunctivitis can rarely be observed clinically. Isolation of fungi from normal conjunctival sac occurs in 6 to $25 \%$ of normal individuals. A seasonal increase in isolation of fungi from the conjunctival sac is observed to occur. This is possibly due to airborne carriage of Candida. Rhinosporidiosis seems to be endemic in the Indian subcontinent.(3-5)

\author{
The Predisposing Factors are.(6) \\ 1. Shared cosmetics. \\ 2. Chronic use of topical broad-spectrum antibiotics. \\ 3. Prolonged use of oral or topical steroids. \\ 4. Injury from vegetable matter. \\ 5. Bathing in stagnant water. \\ 6. Immuno-compromised patients.
}

Common fungi causing conjunctivitis are: Candida albicans, Candida parapsilosis, Candida tropicalis, Paracoccidioides brasiliensis, Coccidioides immitis, Blastomyces dermatitidis, Rhinosporidium seeberi, Sporothrix schenckii, etc.

\section{Clinical Features}

\section{These are:}

1. Redness.

2. Itching.

3. Discharge.

4. Irritation.

There is chronic inflammation and scanty eye discharge. The conjunctiva is congested with oedema formation. Granulomas can be seen.(7) The discharge may be yellow or green in colour.

Candida conjunctivitis commonly occurs in newborn, school children and adults with the primary lesion being in oral mucosa or vagina. Conjunctival membrane or pseudomembrane may occur.

Malassezia infection presents with catarrhal conjunctivitis, whereas Coccidioides immitis causes a severe necrotising granular conjunctivitis and sometimes follicular conjunctivitis. Blastomyces dermatitidis causes contiguous spread and follicular conjunctivitis. Sporothrix schenckii presents with nodular conjunctivitis along with associated deep lesions and lymphadenopathy. Aspergillus niger causes chronic conjunctivitis with black conjunctival secretions. ${ }^{(8,9)}$

Immunocompromised patients may experience a severe clinical course with granulomatous conjunctivitis or necrotising conjunctivitis with scleral melting. The condition may masquerade as squamous cell carcinoma, a typical papilloma or conjunctival granuloma. ${ }^{(9)}$

Biopsy and histopathology is the recommended diagnostic procedure. Giemsa stain of conjunctival scrapings may demonstrate the presence of small intracellular or extracellular yeasts. Specific culture or PCR has a more diagnostic yield.

Topical antifungal agents may be used. These include Amphotericin B (0.15\%), Natamycin (5\%), Fluconazole (2\%) and Ketoconazole (2\%). Systemic antifungals may be used for associated deep lesions.
Necrotising granulomatous conjunctivitis due to Coccidioides immitis requires aggressive debridement of the affected area and prolonged topical Amphotericin B and oral fluconazole therapy. Blastomycosis and Sporotrichosis are treated with itraconazole. Rhinosporidium seeberi presents with fleshy, friable, red pedunculated mass which must be excised with adequate margin.(7) Medical treatment is of no value in these cases.

\section{UVEA, Vitreous and Retina}

Fungi may cause uveitis, vitritis, retinitis, chorioretinitis and even endophthalmitis. All these disease entities may hamper vision to a significant extent, if not treated on time.

Presumed Ocular Histoplasmosis Syndrome (POHS) is a syndrome characterised by multiple scars in the choroid (Histo spots), neovascularization, peripapillary atrophy with a clear vitreous and aqueous. POHS affects young and middle aged adults and is a major cause of choroidal neovascularisation in patients younger than fifty years. POHS causes permanent loss of vision in many patients. It is associated with HLA-DR15 (A subtype of HLA-D2) and HLADQ6 haplotype. (10) While rare cases of fungal endophthalmitis have been seen in patients with disseminated histoplasmosis, the association of Histoplasma capsulatum with POHS is presumptive and based primarily on epidemiologic grounds. In the United States POHS is most prevalent in the Midwest, which is a region endemic for histoplasmosis. A previous study in 1972 found that $4.4 \%$ of people living in an endemic area, who had tested positive by a histoplasmin skin test had ocular histo spots.(11) Other support for histoplasma capsulatum as the aetiology is lacking. However, POHS has been found in areas where $\mathrm{H}$. capsulatum is absent such as the Netherlands.(12) Laser photocoagulation and intravitreal administration of anti-VEGF agents are the primary treatment modalities. There is no indication for antifungal treatment.

\section{Fungal Endophthalmitis}

Endophthalmitis is an inflammatory process involving the ocular cavity and adjacent structures. Infectious endophthalmitis is mainly caused by fungus and bacteria. It is increasingly being understood that virtually any microorganism can cause an infection inside the eye, if the inoculum is a very large one. The most common fungus, which can cause metastatic endophthalmitis is Candida spp., especially in the setting of active fungaemia.

Based on the mode of entry of the fungus, the endophthalmitis is divided into exogenous and endogenous types. Exogenous infection occurs when the fungus is introduced into the human eye from the environment. Endogenous endophthalmitis occurs when haematogenous spread of fungus occurs.

Based on aetiological type of microorganism, the endophthalmitis can be bacterial type of infection or fungal type of infection. Exogenous infection occurs when the fungus gains entry from outside. Endogenous endophthalmitis occurs if the disease is caused by the entry of fungi through the blood stream (Haematogenous route).

Amongst the exogenous endophthalmitis, $70 \%$ are postoperative endophthalmitis, which may be an early or late onset. Penetrating ocular trauma accounts for $7-30 \%$ or cases. Early onset endophthalmitis due to fungus may occur within 2 to 7 days after surgery, but later onset is commoner. 
The value of culture of vitreous and aqueous samples in the diagnosis of infectious endophthalmitis is well established. Sometimes negative cultures are encountered resulting in a clinical dilemma over the cause of the inflammation. It is difficult clinically to differentiate between infectious endophthalmitis and other similar inflammatory conditions, such as phacoanaphylactic uveitis and idiopathic postoperative inflammation.

\begin{tabular}{|c|c|c|}
\hline \multicolumn{2}{|c|}{$\begin{array}{c}\text { Category of } \\
\text { Endophthalmitis }\end{array}$} & $\begin{array}{c}\text { Common Causative } \\
\text { Organism }\end{array}$ \\
\hline Postoperative & Acute & \multirow{3}{*}{ Candida spp. } \\
\hline & Delayed & \\
\hline & $\begin{array}{l}\text { Filtering } \\
\text { bleb }\end{array}$ & \\
\hline Post-traumatic & Fungal & $\begin{array}{c}\text { Penicillium spp., } \\
\text { Fusarium spp., } \\
\text { Acremonium spp., } \\
\text { other filamentous fungi }\end{array}$ \\
\hline Endogenous & Fungal & $\begin{array}{l}\text { Yeasts (C. albicans, } \\
\text { Cryptococcus spp.), } \\
\text { Filamentous fungi } \\
\text { (Aspergillus spp., } \\
\text { Acremonium, } \\
\text { Fusarium, } \\
\text { Paecilomyces spp.) }\end{array}$ \\
\hline Table 2: Fu & Aetiolo & f Endophthalmitis \\
\hline
\end{tabular}

Chronic endophthalmitis occurs late due to less virulent organisms like fungi, S. epidermidis and P. acne. Fungal endophthalmitis may also be caused by saprophytes or opportunistic pathogens (e.g., Cephalosporium, Candida, Aspergillus or Penicillium). Bleb induced endophthalmitis occurring after trabeculectomy operation is usually caused by virulent bacteria.

Endogenous endophthalmitis is typically caused by fungi, e.g. Candida parapsilosis, C. krusei and C. stellatoidea. The next causative fungus in order of occurrence is Aspergillus spp. of which Aspergillus fumigatus dominates, but A. flavus has also been found. Some other fungi like Sporothrix schenckii, Cryptococcus neoformans, C. immitis and Mucor have been reported in isolated cases.

In the laboratory diagnosis of the causative agents of infection, the vitreous is the most appropriate specimen. It is subjected to smear and staining and is also cultured in SDA and PDA media.

Culture of non-ocular specimens is very significant for the diagnosis of endogenous endophthalmitis. The first important step is blood culture. Sputum culture is important in cases of respiratory tract infection (both upper and lower), which may be caused by fungi. Stool culture is important for gastrointestinal infections. Cerebrospinal fluid is cultured in cases of suspected meningitis. Other occult sites which must be explored in a case of endogenous endophthalmitis are bone marrow (biopsy to be taken) and liver (biopsy to be taken). Molecular biological techniques may be used to detect Candida albicans.

The treatment is by vitrectomy with intravitreal fungal antibiotics like Amphotericin B, which gives encouraging results only when performed on time. The prognosis is always guarded as most eyes become phthisical with no vision.
The patients may be divided into two major categories by incidence and response to therapy. These two categories are Candida endophthalmitis and mould endophthalmitis. In industrialized nations and cooler climates, Candida endophthalmitis is commoner than mould endophthalmitis. The reverse is true in tropical countries.

Candida endophthalmitis is usually endogenous and responds well to treatment, whereas mould endophthalmitis is almost always exogenous and successful therapy is uncommon. Endophthalmitis due to Cryptococcus and the dimorphic fungi Histoplasma and Coccidioidomycosis is rare and almost always a result of disseminated disease.

Endophthalmitis due to moulds is rare in western countries, but it is seen in tropical parts, e.g. Florida in USA where $6 \%$ of 278 endophthalmitis cases treated between 1996 and 2001 were due to Aspergillus and other moulds.

In tropical countries such as India, fungal endophthalmitis is a significant problem. Moulds accounted for $22 \%$ of 170 postoperative endophthalmitis cases in Northern India.(13) and $21 \%$ of 170 postoperative endophthalmitis cases in Southern India.(14)

Mould endophthalmitis is usually exogenous with most cases occurring as an extension of fungal keratitis (Keratomycosis) after penetrating eye trauma or after eye injury.(15) in intravenous drug abusers and in immunocompromised patients including organ transplant patients and patients with haematologic malignancies. $(16,17,18)$ Most of these immunocompromised patients have a focus of fungal infection elsewhere, usually the lungs.(16,17) Mould endophthalmitis developing after surgery usually presents subacutely 2-6 weeks postoperatively. Aspergillus is the common cause of mould endophthalmitis causing $50 \%$ to $60 \%$ of cases. $(14,19,18)$ Fusarium is another common cause of mould endophthalmitis and most cases result from Fusarium keratitis.(20) Several cases occurred after a nationwide outbreak of Fusarium keratitis from 2004 to 2006 associated with a contact lens cleaning solution.(21)

\section{Chorioretinitis and Vitritis}

Rarely, fungi such as Coccidioides spp., Blastomyces spp., Aspergillus spp. and Cryptococcus neoformans may cause chorioretinitis by haematogenous spread. Nocardia spp. can occasionally cause chorioretinitis. Candida spp. can cause retinitis and vitritis in neonates. The risk factors are prematurity, low birth weight, sepsis, malnutrition and treatment with broad-spectrum antibiotics. Opportunistic infections with Pneumocystis jirovecii, H. capsulatum, C. neoformans may produce multifocal choroiditis in patients who have Acquired Immunodeficiency Syndrome (AIDS).

\section{Treatment}

Intravitreal antifungal agents are given. Some cases need vitrectomy. The underlying disease process must be controlled. The overall visual prognosis is guarded with useful vision being retained only when treatment is started early.

\section{Face, Paranasal Sinus, Orbit and Ocular Adnexa}

The usual presentation in these organs is a fungal cellulitis. This can be very difficult to treat and may cause death if not treated properly.(22-24) Immunocompromised status due to uncontrolled diabetes mellitus, steroid abuse, drug abuse or HIV infection may cause uncontrollable infection and 
death. $(24,25,26)$ In diabetic patients, proper management of the systemic metabolic parameters are very important. $(27,28)$

Fungal facial cellulitis is a rare entity and unfortunately has ill-defined treatment guidelines. A high index of clinical suspicion in appropriate clinical settings is essential for early diagnosis and successful outcome. Bodenstein NP et al mentioned that a black eschar on palate, nose or orbit is a definite clue to the diagnosis. Nontender, periorbital oedema is an early feature due to orbital involvement.(29) The initial symptoms of rhinocerebral mucormycosis may be eye pain, facial pain or facial numbness followed by onset of conjunctival oedema, blurred vision and soft tissue swelling.(30)

Dhiwakar $\mathrm{M}$ et al(31) report that perinasal cellulitis or paraesthesia is the most common early sign. Periorbital oedema, mucopurulent rhinorrhoea and nasal crusting were other early manifestations. The CT scan according to them could be near normal. For immunocompromised patients, therefore a high degree of suspicion is important. Kotzamanoglou $\mathrm{K}$ et al( ${ }^{32)}$ highlighted the need to differentiate between fungal orbital cellulitis and bacterial cellulitis as the management is different in two cases and misdiagnosis can cause severe complications. Davis and Stearns. ${ }^{(33)}$ highlighted the importance of early CT scan in diagnosing sinusitis along with orbital cellulitis, which improved management outcomes. Talmi Y P et al(34) reported that on CT scan, thickening of mucosa of paranasal sinuses or thickening of extraocular muscles were diagnostic. Retro-orbital mass or abscess may be present. If the eyes have no useful vision, enucleation can often be life-saving.(35) Fungal culture reports from pus or excised tissue may take 3 to 7 days to be available. Serological tests or PCR is not very reliable. Microbiologic fungi demonstration is controversial, because Mucor is a commensal and a common laboratory contaminant.

Histopathological studies reveal that in tissue Mucorales hyphae can be distinguished from common opportunistic moulds by their broad (3-25 $\mu \mathrm{m}$ ) diameter thin walled, mostly aseptate hyphae together with angio-invasion.

Amphotericin B is the drug of choice. The recommended dose of amphotericin B deoxycholate is 1 to $1.5 \mathrm{mg} / \mathrm{kg} /$ day. ${ }^{36-}$ 39) Surgical debridement and antifungal therapy carries the best prognosis. Monotherapy with antifungal agents are ineffective due to poor penetration of these agents into necrotic tissues, which have poor blood supply. Local application of antifungal agents has a poor response. Extensive debridement of deeper tissues is the only available life-saving treatment.(40) Orbital exenteration may be required as well as paranasal sinus excision and they seem to work by reducing the infective load.(40,41) However posaconazole, an orally available broad-spectrum triazole $(800 \mathrm{mg}$ per day in divided doses) appears to have clinically useful activity against several species of Mucorales. $(42,43)$

Cost of therapy may limit the proper drug delivery (by liposomal amphotericin). Early diagnosis by the Microbiology Laboratory with Gram stain, potassium hydroxide wet mount and the use of special stains are essential. A contrast CT scan/MRI scan of face, PNS, orbit and brain should be done whenever deeper infection is suspected. Therapeutic measures should include surgery as well as drug therapy with the most effective antifungal agents, so as to be lifesaving.

\section{CONCLUSION}

Fungal ocular infections thus present with diverse clinical pictures in addition to keratomycosis. These disease entities are common and are sometimes difficult to treat. If untreated or poorly treated, many of these conditions can become vision threatening or even life-threatening. Thus, accurate and rapid diagnosis and adequate treatment are extremely important in fungal ocular infections. The common aetiological factors include presence of uncontrolled diabetes mellitus, use of over-the-counter steroid preparations, haematologic malignancies, staying in areas where fungus infection is endemic and immunocompromised patients. Many of these factors are correctable, treatable and preventable. With many new antifungal agents on the horizon, e.g. posaconazole, voriconazole, the future of these patients are brighter than previous days. Newer treatment modalities and antifungal agents are undergoing trials and there is definite hope that at least some fungal diseases will be eradicated in the future.

\section{REFERENCES}

1. Chakraborty A, Chatterjee SS, Shivaprakash MR. Overview of opportunistic fungal infections in India. Nihon Ishinkin Gakkai Zasshi 2008;49(3):165-72.

2. Gunasekaran S. Approach to a case of infectious keratitis. DOS Times 2013;19(1):37.

3. Klotz SA, Penn CC, Negvesky GJ, et al. Fungal and parasitic infections of the eye. Clin Microbiol Rev 2000;13(4): 66285.

4. Ando N, Takatori K. Fungal flora of the conjunctival sac. Am J Ophthalmol 1982;94(1):67-74.

5. Segal E, Romano A, Eylan E, et al. Fungal flora of the normal conjunctival sac. Mykosen 1977;20(1):9-14.

6. Behrens-Baumann W. Developments in ophthalmology: mycosis of the eye and its adnexa. Switzerland Karger 1999;32:70.

7. Barnes SD, Pavan-Langston D, Azar DT. Microbial conjunctivitis. In: Mandell GL, Bennett DR, editors. Principles and practice of infections diseases. $7^{\text {th }}$ edn. Philadelphia: Churchill Livingstone Elsevier 2010: 1529-37.

8. Sehgal S, Dhawan S, Chibber S, et al. Frequency and significance of fungal isolation from conjunctival sac and their role in ocular infections. Mycopathologia 1981;73(1):17-9.

9. Ben Ezra David. Blepharitis and conjunctivitis. Guidelines for diagnosis and treatment. $1^{\text {st }}$ ed. Israel, Editorial Glosa 2006:104-5.

10. Humeyra D, Kaplan HJ, Duffy BF, et al. Association of the HLA-DR $15 / \mathrm{HLA}^{-\mathrm{DQ}_{6}}$ haplotype with development of choroidal neovascular lesion in presumed ocular histoplasmosis syndrome. Human Immunology 2003;64(10):960-4.

11. Smith RE, Ganley JP. Presumed ocular histoplasmosis. I. Histoplasmosis skin test sensitivity in cases identified during a community survey. Arch Ophthalmol 1972;87(3):245-50.

12. Suttop-Schulton MSA, Bollemeijer JG, Bos PJM, et al. Presumed ocular histoplasmosis in the Netherlands-an area without histoplasmosis. Br J Ophthalmol 1997; 81: 7-11. 
13. Gupta A, Gupta V, Gupta A, et al. Spectrum and clinical profile of post cataract surgery endophthalmitis in North India. Indian J Ophthalmol 2003;51(2):139-45.

14. Anand AR, Therese KL, Madhavan HN. Spectrum of aetiological agents of postoperative endophthalmitis and antibiotic sensitivity of bacterial isolates. Indian J Ophthalmol 2000;48(2):123-8.

15. Garg P, Mahesh S, Bansal AK, et al. Fungal infection of sutureless self-sealing incisions for cataract surgery. Ophthalmology 2003;110(11):2173-7.

16. Narang S, Gupta A, Gupta V, et al. Fungal endophthalmitis following cataract surgery: clinical presentation, microbiological spectrum and outcome. Am J Ophthalmol 2001;132(5):609-17.

17. Chakraborti A, Shivaprakash MR, Singh R, et al. Fungal endophthalmitis: 14 years' experience from a centre in India. Retina 2008;28:1400-07.

18. Lamaris GA, Esmaeli B, Chamilos G, et al. Fungal endophthalmitis in a tertiary care cancer centre: a review of 23 cases. Eur J Clin Microbiol Infect Dis 2008;27(5):343-7.

19. Wykoff CC, Flynn HW, Miller D, et al. Exogenous fungal endophthalmitis: microbiology and clinical outcomes. Ophthalmology 2008;115(9):1501-7.

20. Rosenberg KD, Flynn HW, Alfonso EC, et al. Fusarium endophthalmitis following keratitis associated with contact lenses. Ophthalmic Surg Lasers Imaging 2006;37(4):310-3.

21. Grant GB, Fridkin S, Chang DC, et al. Post-recall surveillance following a multistate fusarium keratitis outbreak, 2004 through 2006. JAMA 2007;298(24): 28678.

22. Yohai RA, Bullock JD, Aziz AA, et al. Survival factors in rhino-orbito-cerebral mucormycosis: major review. Surv Ophthalmol 1994;39(1):3-22.

23. Parfrey NA. Improved diagnosis and prognosis of mucormycosis: a clinicopathologic study of 33 cases. Medicine 1986;65(2):113-23.

24. Blitzer A, Lawson W, Meyers BR, et al. Patient survival factors in paranasal sinus mucormycosis. Laryngoscope 1980;90(4):635-48.

25. Morais PD, Guerra GA, Alonso JB, et al. Rhino-orbitocerebral mucormycosis. Review, update and report of new case. Acta Otorrhinolaringol Esp 1997;48(4):309-13.

26. Marchershy AM, Bottone EJ, Geller SA, et al. The changing spectrum of disease, aetiology and diagnosis of mucormycosis. Hum Pathol 1980;11(5):457-64.

27. Abramson E, Wilson D, Arky RA. Rhino cerebral phycomycosis in association with diabetic ketoacidosis. Ann Intern Med 1984:1060-2.

28. Artis WM, Fountain JA, Delcher HK. A mechanism of susceptibility to mucormycosis in diabetic ketoacidosis: transferrin and iron availability. Diabetes 1982;31(12):1109-14.
29. Bodenstein NP, McIntosh WA, Vlantis AC, et al. Clinical signs of orbital ischaemia in rhino-orbito-cerebral mucormycosis. Laryngoscope 1993;103(12):1357-61.

30. Spellberg B, Edwards J, Ibrahim A. Novel perspectives on mucormycosis: pathophysiology, presentation and management. Clin Microbiol Rev 2005;18(3):556-69.

31. Dhiwakar M, Thakar A, Bahadur S. Improving outcomes in rhinocerebral mucormycosis-early diagnostic pointers and prognostic factors. J Laryngol Otol 2003;117(11):861-5.

32. Kotzamanoglou K, Tzanakakis G, Michelopoulos E, et al. Orbital cellulitis due to mucormycosis: a case report. Graefes Arch Clin Exp Ophthalmol 1988;226(6):539-41.

33. Davis JP, Stearns MP. Orbital complications of sinusitis: avoid delays in diagnosis. Postgrad Med J 1994;70(820):108-10.

34. Talmi YP, Goldschmied-Reouven A, Bakon M, et al. Rhinoorbital and rhino-orbito-cerebral mucormycosis. Otolaryngol Head Neck Surg 2002;127(1):22-31.

35. Peterson KL, Wang M, Canalis RS, et al. Rhinocerebral mucormycosis: evolution of the disease and treatment options. Laryngoscope 1997;107(7):855-62.

36. Drogari-Apivanthilon M, Mantopoulou FD, Skiada A, et al. In vitro antifungal susceptibility of filamentous fungi causing rare infections: synergy testing of amphoterion $\mathrm{B}$, posaconazole and anidulafungin in pairs. J Antimicrob Chemother 2012;67(8):1937-40.

37. Salas V, Pastor FJ, Calvo E, et al. In vitro and in vivo activities of posaconazole and amphotericin $B$ in a murine invasive infection by mucor circinelloides: poor efficacy of posaconazole. Antimicrob Agents Chemother 2012;56(5):2246-50.

38. Krishnan-Natesan S, Manavathu EK, Alongadu GJ, et al. A comparison of the fungicidal activity of amphotericin B and posaconazole against zygomycetes in vitro. Diag Microbiol Infect Dis 2009;63(4):361-4.

39. Wildfeuer A, Seidl HP, Paule I, et al. In vitro evaluation of voriconazole against clinical isolates of yeasts, moulds and dermatophytes in comparison with itraconazole, ketoconazole, amphotericin B and griseofulvin. Mycoses 1998;41(7-8):308-19.

40. Odvi JW, Harris JP, Feldman JI, et al. Rhinocerebral mucormycosis: results of aggressive surgical debridement and amphotericin B. Laryngoscope 1998;98(12):1339-42.

41. Luna JD, Ponssa XS, Rodriguez SD, et al. Intraconal amphotericin B for the treatment of rhino-orbital mucormycosis. Ophthalmic Surg Lasers 1996;27(8): 7068.

42. Mohindra S, Mohindra S, Gupta R, et al. Rhinocerebral mucormycosis: the disease spectrum in 27 patients. Mycoses 2007;50(4):290-6.

43. Malis A, Mastronikolis SN, Naxakis SS, et al. Rhinocerebral mucormycosis: an update. Eur Rev Med Pharmacol Sci 2010;14(11):987-92. 